\title{
Turismo sexual: \\ saunas para varones \\ en la ciudad de Puebla
}

DOI: 10.22403/UQROOMX/TYP05/08

$\Delta$

Mauricio List Reyes
Alberto Teutle López

\section{Introducción}

En México se ha experimentado un creciente interés por la investigación en torno a la diversidad sexual, siendo importante el análisis de variadas prácticas sociales y de los espacios en las que se llevan a cabo, lo cual ha permitido comprender las formas complejas en las que se da el uso del espacio y las interacciones sociales que permiten. Incluso, algunos de estos estudios se han centrado en el examen de los propios sitios y en los itinerarios que a partir de ellos se establecen.

Así, la Antropología, la Historia, la Sociología y la Geografía, entre otras disciplinas, han posibilitado acercarse a un sólido estudio de estos lugares en contextos fundamentalmente urbanos. Se han realizado investigaciones tanto etnográficas como geográficas con aportes significativos para la comprensión no sólo de esos sectores en particular, sino también del uso del espacio urbano y el desarrollo de las metrópolis (Delgado, 2002: 125).

Por fortuna, cada vez son más las investigaciones que se están realizando en el país, con un notable incremento en ciudades medias. Un punto central ha sido comprender las dinámicas urbanas de los sectores sociales analizados: sus itinerarios, sus rutas y su acceso a los espacios urbanos, donde en múltiples ocasiones tienen que enfrentarse con una abierta y explícita homofobia de los dueños, empleados y clientes de estos sitios.

*Benemérita Universidad Autónoma de Puebla, Colegio de Antropología Social / maurilist@yahoo.com.mx / chigripo@yahoo.com 
En los últimos cinco años se ha investigado el uso que sujetos sexodiversos hacen de los espacios urbanos en Puebla; se examina la forma en que se construyen los itinerarios en el centro histórico, cómo se han dado los procesos de apropiación de diversos lugares, la construcción de las identidades de hombres y mujeres, el papel del consumo en la organización del espacio entre clases sociales, etcétera.

En este trabajo se analizan las dinámicas urbanas de los varones que acuden a distintos saunas de la capital poblana, en las que participan hombres provenientes de diferentes estados del país y que, por diversas razones, han elegido esta ciudad como punto de destino o de escala en su tránsito a otras entidades. En este trabajo se documenta particularmente la presencia de quienes llamamos turistas sexuales: sujetos varones cuya actividad principal, o una de las principales, al escoger a la ciudad de Puebla, es acudir a varios espacios de encuentro tanto para socializar como para ejercer su sexualidad. Por ello, nos hemos enfocado en cuatro recintos de sauna: dos baños públicos y dos saunas que sirven como sitios de encuentro para varones gay.

\section{Antecedentes}

Históricamente, la ciudad de Puebla ha sido un punto estratégico en la geografía regional del país. Su ubicación -como punto intermedio entre la capital y el puerto de Veracruz, por un lado, y la conexión que tiene con el sureste mexicano, por el otro- la convirtió en escala obligada de muchos trayectos. Por esta razón, no debe perderse de vista que el flujo de visitantes suele ser muy alto a lo largo del año, al margen de los periodos vacacionales.

Puebla es una de las ciudades más importantes del centro del país que aglutina una amplia oferta educativa de nivel superior y una planta industrial que emplea a gran cantidad de trabajadores. Esto ha provocado una elevada inmigración temporal de jóvenes en busca de esa oferta educativa o de empleo, lo cual ha estimulado una alta concentración de personas originarias de las distintas localidades del estado, así como de otras entidades del sureste.

En el contexto regional, la ciudad de Puebla también es un enclave muy significativo, a pesar de existir poblaciones tan representativas como Tehuacán, Tlaxcala, Apizaco, Atlixco, San Martín Texmelucan, entre otras. 
La elevada movilidad ha provocado que sea reconocida no sólo como una ciudad media de enorme peso regional, sino que para muchos constituya un sitio ideal para acceder a una oferta de encuentros sexuales clandestinos en un contexto que ofrece la posibilidad de pasar inadvertido; esto, bajo los conceptos en los que es pensada la ciudad en sus relaciones superficiales y anónimas, como lo anota Manuel Delgado (2002: 25).

En Puebla, la oferta de servicios para la población sexodiversa ha crecido en la última década, lo cual hizo visible a un sector muy significativo, principalmente de jóvenes, quienes abarrotan cada fin de semana los bares y discotecas de la ciudad dirigidos a diferentes sectores socioeconómicos (List, 2007: 198).

Aunado a ello, se creó una oferta de puntos de encuentro que permiten el ejercicio de la sexualidad. De este modo, se abrieron dos espacios en distintas zonas de la ciudad para dos sectores socioeconómicos: uno para un sector medio y el otro para un sector bajo, pero en ambos casos la oferta es en esencia para sujetos que se reconocen a sí mismos como gays.

A su vez, existe otra oferta dirigida a sujetos que no se asumen como sexodiversos; de hecho, estos recintos son resultado de un lento proceso de apropiación del espacio por parte de individuos que requerían un lugar en el que pudieran tener encuentros sexuales de forma clandestina, pero que además fuera lo suficientemente discreto para evitar cualquier clase de sospecha acerca de su sexualidad. En ese aspecto, se trata de sitios que han adquirido esta característica aun a pesar de sus dueños y empleados, y cuyos clientes aprovechan tanto lo económico como lo accesible de los lugares para tener esa clase de encuentros (Teutle, 2007: I5).

Es justo a estas ofertas de servicios a las que se refiere este escrito: los espacios que son aprovechados por sujetos de diverso nivel socioeconómico que realizan turismo en la ciudad de Puebla, procedentes de varios puntos nacionales e internacionales, y que, por diferentes motivos, prefieren este tipo de lugares.

\section{Los saunas en Puebla}

Es importante señalar que en Puebla los baños públicos tienen una larga historia, se extienden no sólo por la ciudad capital sino por poblaciones cercanas. Incluso, en muchas comunidades y barrios es frecuente ver a familias completas que 
acuden el fin de semana a compartir el espacio del baño. Al terminar, las mujeres suelen salir con la toalla enrollada en la cabeza, $y$ todos los miembros de la familia calzan sandalias y cargan cubetas con prendas y artículos de baño húmedos.

Algunos de estos baños se encuentran en antiguas construcciones de más de una centuria, que han permanecido a pesar de las transformaciones urbanas y de los embates de la modernidad; pero ofrecen una imagen decadente, incluso sucia, lo que no ha impedido que sean visitados por clientes que han descubierto ahí la posibilidad de acercarse a individuos como ellos y de obtener placer de otro cuerpo masculino.

Sin embargo, ésta no es toda la oferta, pues habría que mencionar los sitios que se construyen expresamente para atender a una población que busca encuentros sexuales entre varones, debido a que cada vez más sitios públicos que solían servir para el encuentro entre sujetos gay han ido restringiendo esas posibilidades al establecer un mayor control:

La vida barroca de estos habitués de las teteras, en todo Occidente, va declinando con la privatización del circuito del sexo en las grandes ciudades y con los cambios arquitectónicos urbanos, durante el salto modernizador del neoliberalismo. Los brillantes espacios interiores de los toilettes de los shoppings y los reconvertidos baños de estación permiten hoy menos escondites para el coito y están siempre vigilados [Rapisardi y Modarelli, 200 I: 22].

En esta investigación se estudiaron cuatro saunas: dos creados para varones gay, $y$ dos que se los han apropiado los hombres a quienes les gusta tener encuentros sexuales entre varones en lugares clandestinos.

El más famoso de ellos, Las Termas, se estableció hace algunos años en la capital del estado, causando furor entre numerosos sujetos gay no sólo de esta urbe, sino también de la Ciudad de México. Desde que se inauguró, su rápida difusión en medios especializados despertó una gran curiosidad, sobre todo porque en la capital del país no existía un sitio con este concepto. Gran parte del éxito de este lugar se ha debido a que sus visitantes lo consideran limpio, seguro, accesible y con un horario muy amplio, ya que los fines desemana funciona las 24 horas del día. Asimismo, el costo es relativa mente moderado, lo que permite convocar a una vasta asistencia, pero a la vez mantiene cierta exclusión de los sectores populares, en buena medida por los precios de su servicio y de las bebidas que ahí se ofrecen. 
Otro sitio también dirigido a la población gay, aunque con menos difusión, es El Ático, un establecimiento en constante remodelación y con un aspecto no muy grato. Se trata de un lugar que permanece en una situación de semiclandestinidad dado que se encuentra ubicado en una de las zonas más populares y limítrofes de la ciudad, lejos de la oferta existente en la urbe para los varones gay. Se localiza muy cerca de la Central de Autobuses de Puebla (Capu), atrae a un público distinto por su condición de clase, pero también porque aquí llegan a mezclarse sujetos gay y otros que no se reconocen como tales. Creado con la intención de servir para el encuentro sexual, su clientela acude con este propósito.

En contraste, hay dos sitios que los sujetos se han apropiado por el uso cotidiano de sus servicios: dos baños sauna públicos, situados también en colonias populares y que originalmente dieron servicio a los habitantes de cada uno de esos barrios, pero al paso del tiempo han sufrido una importante transformación, básicamente en las salas de vapor colectivo.

Uno de ellos se encuentra a un costado de uno de los mercados más grandes de una concurrida zonas comercial de la ciudad y de la región, y también cerca de la Central de Autobuses, por lo que muchos de sus usuarios son tanto clientes como comerciantes del mercado y de las zapaterías y bodegas que venden al mayoreo. A pesar de encontrarse ubicado en una pequeña colonia, son pocos los vecinos varones que acuden a él, en gran medida por su fama de ser un punto de encuentro sexual para hombres.

El otro sauna está cerca de la encrucijada que forman dos importantes bulevares que atraviesan buena parte de la ciudad. Es una zona con intensa carga vehicular y una gran circulación de transporte público, lo que facilita el acceso y no ser advertidos, lo cual para muchos es una ventaja frente a los otros sitios aquí mencionados.

Estos establecimientos prestan sus servicios convencionales; sin embargo, la sala de vapor colectivo se ha modificado para permitir la práctica sexual con la complicidad de los empleados, quienes se mantienen al margen.

A pesar de su origen y su dinámica cotidiana, estos espacios empiezan a ser frecuentados por hombres gay, cuyas prácticas sexuales son diferentes de las de los clientes regulares. 
Cabe señalar que los saunas públicos como puntos de encuentro para el sexo entre varones no son privativos de la ciudad de Puebla, son espacios que existen tanto en el resto del país como en muchas capitales del mundo. De hecho, un elevado número de guías de viajes especializadas en turismo gay, así como publicaciones dirigidas a ese sector, señalan como puntos de encuentro los saunas públicos, que cada día se vuelven más refinados y especializados, con una multiplicidad de servicios no sólo para el encuentro sexual sino para hacer grata la estancia de los clientes, aun de quienes únicamente buscan un ambiente gay para relajarse y sentirse cómodos.

\section{Los viajeros sexuales}

Es pertinente señalar un aspecto que marca a todos los sujetos a los que se hace referencia en este trabajo: la masculinidad. No obstante, estamos hablando de sujetos que han construido esa masculinidad de manera diversa; los discursos en torno a ésta se mantienen y a la vez se traducen de forma distinta, en lo cual pueden incidir otros elementos que aquí podríamos sintetizar como homofobia y clóset. Sin duda, estos aspectos no son los que determinan el uso de cierto tipo de espacios para el encuentro sexual y para socializar, pero sí marcan en buena medida la actitud de los sujetos que participan en ellos (Guasch, 2000: 65).

Es importante tomar en cuenta que los visitantes de estos vapores son, en la mayoría de los casos, originarios de otras entidades del país o de otras ciudades del propio estado.A pesar de que ahí llegan tanto hombres gay como hombres que tienen sexo con hombres, sin establecer una identidad a partir de esa práctica sexual, se mantiene una diferenciación tácita entre los sitios que suelen frecuentar unos y otros.

Habría entonces que diferenciar a ambos sujetos para entender las razones que los llevan a acudir a esos lugares: los gay suelen frecuentar los sitios creados ex profeso, mientras que los hombres que gustan de tener esa clase de encuentros sin las implicaciones de asumir una identidad frecuentan los espacios clandestinos.

A los sujetos gay y bisexuales que suelen acudir a estos sitios los mueve un interés por descubrir la variedad de ofertas tanto institucionalizadas como clandestinas, que han venido promocionándose entre los sujetos gay sobre todo a través de las páginas web o de las recomendaciones que hacen entre sus pares. 
Cabe apuntar que en los negocios referidos en esta investigación no se ejerce el sexoservicio, lo cual se ha podido constatar a partir del trabajo de campo realizado en diversas temporadas, ya que los individuos que ahí se reúnen no están interesados en ofrecer servicios sexuales ni en contratarlos.

Asimismo, se ha comprobado que en algunos de estos recintos se prohíbe expresamente el sexoservicio, y en ocasiones se invita a los asistentes a denunciar este tipo de prácticas.

Lo que sí se ha observado es lo que en la jerga de estos sectores sociales se denomina chichifeo, es decir, solicitar y recibir alguna dádiva en especie-que puede ir desde una cerveza o una invitación a comer, hasta una prenda de ropa o algo semejante- a cambio de compañía e incluso de algún contacto sexual.

Por ejemplo, en uno de los saunas públicos trabaja un masajista joven y atractivo que es reconocido por los clientes como heterosexual, quien además de dar servicios de masaje continuamente juega con los clientes, los manosea o tiene con ellos alguna otra clase de contacto físico, lo que por lo regular es retribuido con invitaciones de cervezas, terminando su día en un ligero estado de ebriedad. Los clientes que lo conocen saben que ellos no pueden iniciar esos contactos con él, pero sí dejar que él lo haga y permitirle que los toque. En uno de los sitios gay aquí mencionados, algunos jóvenes, conscientes del potencial de estas actividades, "se dejaban querer" por los visitantes, sobre todo por aquellos que acudían por única ocasión. Así, era común ver a estos jóvenes pasearse continuamente por el lugar, esperando encontrar a algún sujeto adecuado que estuviera dispuesto a participar en ese juego.

Cuerpo y masculinidad tienen una relación que resulta significativa, ya que suele estar atravesada por la preferencia sexual, asunto por demás inquietante para hombres y mujeres en las sociedades contemporáneas; esto es, la manera en que los sujetos perciben y usan su cuerpo (McDowell, 2000: 84).

Descubrimos entonces un abanico de posibilidades en estos baños de vapor: los sujetos que, reconociéndose a sí mismos como heterosexuales, "se sienten calientes y quieren coger con lo que sea"; los que se dicen bisexuales, muchos de ellos casados, que buscan tener encuentros con varones dentro de estos contextos; y los gays, quienes saben que en estos recintos hallarán hombres dispuestos a tener un breve encuentro en el anonimato. Evidentemente, no en todos los casos está reconocida la identidad sexual en los términos aquí planteados. 
Vale la pena señalar que, no obstante que se han creado espacios para atender a un público específicamente gay, es decir, sitios para el encuentro sexual entre varones, en ningún momento esto le quita a los usuarios de los saunas el interés por visitar esta clase de lugares, por lo que los clientes no suelen ser los mismos en ambos casos.

\section{Los encuentros anónimos}

Debe resaltarse el hecho de que los sujetos que acuden a estos sitios, por lo general no se plantean como objetivo principal encontrar o establecer una relación formal con algún usuario de esos lugares.Van sobre todo con la intención de tener encuentros anónimos, lo cual se espera que transcurra con el menor intercambio de información, pues, en la mayoría de los casos, ni siquiera se conoce el nombre de la persona con la que se ha tenido el contacto.

A partir del trabajo de campo desarrollado durante varios años en la capital de Puebla se ha descubierto que muchos sujetos que habitan en ciudades cercanas (San Martín Texmelucan, Tlaxcala, Apizaco, Tehuacán, Atlixco, Tecamachalco), así como en muchas pequeñas localidades de la entidad y de los estados de Tlaxcala,Veracruz y México, acuden a estos recintos para poder tener estos encuentros furtivos.

Una gran cantidad de ellos expresó su enorme temor de ser descubiertos por familiares o amigos, por lo que en múltiples ocasiones tenían que hacer largos recorridos para encontrar un lugar donde no existiera la posibilidad de ser reconocidos.

¿Qué puede resultar atractivo en un sitio como éste? Sin duda, mucho tiene que ver con la homofobia permanente de distintos contextos sociales, $y$, en el caso de numerosos sujetos gay, suele ser importante relacionarse con individuos aparentemente heterosexuales por el gusto de vincularse con alguien "que se vea como hombre". Para otros, el atractivo se funda en el hecho de que es un espacio que no necesariamente tiene esta connotación sexual y, por tanto, no por usar el sitio define a su clientela como gay, como en los saunas ex profeso.

Los hombres que aquí se reúnen han construido una topografía corporal del placer, partes del cuerpo que pueden ser tocadas frente a otras que no lo pueden ser, precisamente porque algunas de ellas son identificadas con lo femenino, sobre todo aquellas que se consideran susceptibles de ser penetradas 
debido a la concepción de lo masculino y lo femenino respecto a la jerarquía de poder (Bourdieu, 2000: 44 y Butler, 200 I: I6).

\section{Hacia dónde apunta la investigación}

Después de este breve recuento de los aspectos centrales de la investigación, se concluye que se trata de un fenómeno complejo, puesto que los procesos de apropiación del espacio se han dado gracias a la asignación de ciertos elementos simbólicos por parte de los sujetos estudiados a partir del uso frecuente. De hecho, esa apropiación pudo ocurrir en la medida en que los sujetos fueron reconociendo las posibilidades que tenían los saunas para permitir los encuentros entre varones; entonces, su continua presencia fue desplazando al resto de los clientes que solían visitarlos; esto es, los usuarios originales han sido reemplazados por sujetos que, más que seguir el ritual de aseo, están a la caza de ejercer su sexualidad con sus iguales.

En ese sentido, la investigación del proyecto de Turismo Sexual en México del Instituto de Geografía de la Universidad Nacional Autónoma de México, permitirá conocer, por un lado, cuáles son esos factores simbólico-culturales que favorecen los procesos de apropiación del espacio, y por el otro, comprender cómo se organiza la actividad turística de los varones que tienen encuentros sexuales con otros varones en los saunas de la ciudad de Puebla. Se pretende impulsar estudios interdisciplinarios para analizar un fenómeno específico: el del uso de los saunas para encuentros sexuales entre hombres, el cual es un fenómeno social distribuido en varias entidades del país.

\section{FUENTES CONSULTADAS}

Bourdieu, Pierre (2000). La dominación masculina. Barcelona:Anagrama.

Butler, Judith (200I). El género en disputa. El feminismo y la subversión de la identidad. México: Paidós/Programa Universitario de Estudios de Género, Universidad Nacional Autónoma de México.

Delgado, Manuel ( 1999). El animal público. Hacia una antropología de los espacios urbanos. Barcelona:Anagrama. 
(2002). Disoluciones urbanas. Procesos identitarios y espacios públicos. Medellín: Universidad de Antioquia/Universidad Nacional de Colombia. Guasch, Oscar (2000). La crisis de la heterosexualidad. Barcelona: Leartes.

List Reyes, Mauricio (2007). "Masculinidades urbanas. Una reflexión a partir de algunos ejemplos de la ciudad de Puebla”, en María Ana Portal (coord.). Espacios públicos y prácticas metropolitanas. México: Universidad Autónoma Metropolitana/Consejo Nacional de Ciencia y Tecnología, I 77-206.

McDowell, Linda (2000). Género, identidad y lugar. Un estudio de las geografias feministas. Madrid: Cátedra/Universidad de Valencia/Instituto de la Mujer.

Rapisardi, Flavio y Alejandro Modarelli (200I). Fiestas, baños y exilios. Los gays porteños en la última dictadura. Buenos Aires: Sudamericana.

Teutle López, Alberto (2007). Húmedos placeres: espacios, género y sexualidad en los varones usuarios de dos baños públicos de Puebla. Tesis de licenciatura en Antropología Social. Puebla: Benemérita Universidad Autónoma de Puebla. 